\title{
Betriebsbedingte Kündigung eines Chefarztes wegen Zentrumsbildung Rationalisierungsmaßnahmen allein reichen als Begründung nicht aus
}

Nicht jede vielleicht aus wirtschaftlichen Gesichtspunkten wünschenswerte Umstrukturierung der Klinikstrukturen ist auch in personeller Hinsicht juristisch haltbar. So kann allein die Tatsache, dass infolge von Rationalisierungsmaßnahmen organisatorische Einheiten zusammengelegt und dadurch Personal abgebaut werden soll, nicht automatisch in eine Kündigung der betreffenden Leitungspersonen münden. Dies hat kürzlich ein Urteil des Sächsischen Landesarbeitsgerichts ergeben. Rechtsgültig ist eine Kündigung in diesem Fall nur, wenn der Arbeitgeber beweisen kann, dass das Bedürfnis zur Weiterbeschäftigung des Arztes in Zukunft tatsächlich entfällt. Für eine rechtsgültige Kündigung muss der Arbeitgeber dafür dem Betriebsrat detailliert offenlegen, in welchem Umfang die bisher vom Arbeitnehmer ausgeübten Tätigkeiten zukünftig anfallen werden. Ein pauschaler Verweis auf den Abbau einer Hierarchieebene und die damit verbundene Neuverteilung der betroffenen Arbeitnehmer beispielsweise reicht nicht für eine sogenannte betriebsbedingte Kündigung aus.

Das Gesundheitswesen ist im Umbruch. Gerade vor dem Hintergrund der zunehmenden Verschlechterung der wirtschaftlichen Situation der gesetzlichen Krankenversicherung (GKV) hat man in der Vergangenheit nach Lösungen gesucht, das System nachhaltig umzugestalten und finanziell zu stabilisieren. Handelte es sich bei diesen Reformen anfangs noch um Versuche, Symptome zu kurieren, so weisen die Reformen der letzten Zeit einen wesentlich anderen Charakter auf: Sie sind echte Strukturreformen. Mit diesen Strukturen aber gehen immer mehr ökonomische Zwänge einher, die den Leistungserbringern auferlegt worden sind. Auch in Zukunft werden sich diesbezüglich sicher noch einige böse Überraschungen ergeben.

\section{Strukturreformen und Zwang zur Effizienzsteigerung}

Insbesondere dem Instrument des Wettbewerbs hat der Gesetzgeber einen hohen Stellenwert beigemessen. So ist es kaum verwunderlich, dass die wirtschaftlichen Zwänge gerade auch Krankenhäuser in einem bisher kaum gekannten Ausmaß treffen. Folge dieser Entwicklung ist das kontinuierliche Bestreben der Kliniken, Wirtschaftlichkeitspotenziale zu erschließen und Kosten zu senken. Häufig geschieht dies durch personelle Veränderungen, aber auch durch die Fusion von Krankenhäusern oder die Übernahme von Konkurrenten. Diese Entwicklung ist deutschlandweit zu verzeichnen und nicht etwa auf einzelne Regionen beschränkt.

Gerade der Krankenhaussektor erlebt daher eine Umstrukturierungswelle in bisher nicht gekanntem Ausmaß. Sie soll dazu dienen, die vorhandenen Ressourcen besser nutzen zu können. Mögen derartige Umstrukturierungsprozesse durchaus von betriebswirtschaftlichem Charme sein, so werden doch die menschlichen Schicksale, die hinter derartigen Entscheidungen stehen, allzu häufig übersehen. Insofern verwundert es auch nicht, wie stark die Zahl arbeitsgerichtlicher Auseinandersetzungen gerade im Zusammenhang mit der Umstrukturierung in Krankenhäusern angestiegen ist.

\section{Arbeitsplatzabbau \\ durch Zentrumsbildung}

Von großer Aktualität ist die beschriebene Problematik insbesondere auch im Zusammenhang mit der Bildung von Zentren oder Instituten, die aus verschiedenen Facheinheiten bestehen und gemeinschaftlich bestimmte Patientengruppen behandeln. Beispielhaft seien etwa die Herzzentren genannt, die verschiedene Bereiche der Herzchirurgie, der Inneren Medizin, aber auch intensivmedizinische Einheiten unter einem Dach vereinen. Erreichen solche Einheiten eine gewisse kritische Größe, werden die angeschlossenen Abteilungen üblicherweise jeweils mit je einem fachlichen Leiter versehen.

Spannend wird es aber dann, wenn im Falle der Bildung eines solchen Zentrums geplant ist, aus 2 ursprünglich eigenständigen Einheiten ein einheitliches Zentrum zu bilden, das mit nur einer Leitungsstelle besetzt ist. Zwangsläufig hat dies das Ausscheiden des der anderen Klinik vorstehenden Chefarztes zur Folge. Wie hoch die Relevanz dieser Frage ist, belegen die zahlreichen Urteile, die mittlerweile hierzu ergangen sind. Jüngstes Beispiel ist eine Entscheidung des Sächsischen Landesarbeitsgerichts, das sich mit der Frage beschäftigt hat, ob ein Krankenhaus einem Chefarzt aus betriebsbedingten Gründen ordentlich kündigen kann, wenn ein solcher Schritt geplant ist (vgl. Urteil vom 07.02.2008 8 Sa 147/07).

\section{Grundsätze der betriebsbedingten Kündigung}

Zunächst scheint es so, dass aus Sicht des Krankenhausträgers die Zusammenführung zweier Einheiten unproblematisch bewerkstelligt werden könnte. Grundsätzlich nämlich ist es allein Sache des Arbeitgebers, wie er seinen Betrieb organisiert. Darunter fallen etwa auch Entscheidungen im Hinblick auf die Organisation der Einrichtung und die Frage, ob und inwieweit Arbeitsplätze gestrichen werden können. Die arbeitsgerichtliche Rechtsprechung hat diesbezüglich jedoch eine Reihe von Voraussetzungen aufgestellt, die für die Wirksamkeit einer sogenannten betriebsbedingten Kündigung einzuhalten sind.

Gilt der allgemeine Kündigungsschutz, so ist eine betriebsbedingte Kündigung durch den Arbeitgeber nur zulässig, wenn 
- dringende betriebliche Erfordernisse einer weiteren Beschäftigung des Arbeitnehmers zu gleichen oder anderen Arbeitsbedingungen entgegenstehen,

- der betroffene Arbeitnehmer von allen vergleichbaren Arbeitnehmern der sozial am wenigsten Schutzwürdige ist und

- eine umfassende (im Ausnahmefall durchzuführende) Interessenabwägung nicht zu dem Ergebnis kommt, dass das Interesse des Arbeitnehmers an der Fortsetzung des Arbeitsverhältnisses gegenüber dem Interesse des Arbeitgebers an dessen Beendigung überwiegt.

\section{Bedürfnis zur Weiterbeschäfti- gung muss entfallen}

In dem bereits erwähnten Urteil des Sächsischen Landesarbeitsgerichts hatte sich das Gericht mit der Frage zu beschäftigen, ob im Falle der Zusammenlegung der bisherigen Kliniken eine betriebsbedingte Kündigung eines Chefarztes zulässig ist. Gegenstand des Sachverhalts, der dem Urteil zugrunde lag, war die Zusammenlegung der bisherigen Kliniken für Orthopädie und Unfallchirurgie zu einem einheitlichen unfallchirurgisch-orthopädischenZentrum mit einem Zentrumsleiter.

Zunächst hatte das Gericht ausgeführt, dass der Chefarzt unzweifelhaft unter die Schutzbestimmungen des Kündigungsschutzgesetzes fällt. Dementsprechend ist also eine Kündigung nur dann möglich, wenn sie sozial gerechtfertigt ist. Dies ist dann der Fall, wenn dringende betriebliche Erfordernisse eine Weiterbeschäftigung des Arbeitnehmers unmöglich machen. Mit anderen Worten: Das Bedürfnis für die Weiterbeschäftigung des Arbeitnehmers muss entfallen sein.

\section{Kündigung nur bei dringenden betrieblichen Erfordernissen}

Nach der ständigen Rechtsprechung des Bundesarbeitsgerichts können sich solche betrieblichen Erfordernisse aus innerbetrieblichen Umständen (Unternehmerentscheidungen wie z.B. Rationalisierungsmaßnahmen) oder durch außerbetriebliche Gründe (z. B. Umsatzrückgang) ergeben. Sie müssen jedoch dringend sein und eine Kündigung im Interesse des Arbeitgebers notwendig machen. Davon ist regelmäßig dann aus- zugehen, wenn die Kündigung unvermeidbar ist, um Schaden vom Unternehmen abzuhalten.

Insofern trägt jedoch der Arbeitgeber im Kündigungsschutzprozess die Beweislast. So hat er vor allem darzulegen, ob das Bedürfnis einer Tätigkeit des gekündigten Arbeitnehmers wegfällt. Das bedeutet, dass inner- und außerbetriebliche Umstände nur dann zu einem dringenden betrieblichen Erfordernis führen, wenn sie sich auch konkret auf die Einsatzmöglichkeit des gekündigten Arbeitnehmers auswirken.

Der Arbeitgeber muss insbesondere erklären, in welchem Umfang die bisherigen vom Arbeitnehmer ausgeübten Tätigkeiten in Zukunft - im Vergleich zum bisherigen Zustand - anfallen werden. Der pauschale Verweis auf den Abbau einer Hierarchieebene und die damit verbundene Neuverteilung der betroffenen Arbeitnehmer reicht hierfür nicht aus. Allein die Zusammenlegung der bisherigen Kliniken zu einem einheitlichen Zentrum mit einem Zentrumsleiter führt nach Ansicht des Landesarbeitsgerichts demnach nicht zum Wegfall der Beschäftigungsmöglichkeit für einen der beiden bisher tätigen Chefärzte.

\section{Beweispflicht beim Arbeitgeber}

Genau hierin liegt aber die häufigste Fehlerquelle für eine betriebsbedingte Kündigung. Zwar betont das Bundesarbeitsgericht immer wieder, dass es im Rahmen der freien unternehmerischen Entscheidung lediglich eine Missbrauchskontrolle ausüben könne. In der Praxis allerdings fällt diese Missbrauchskontrolle in der Regel sehr breit aus. Insbesondere prüfen die Arbeitsgerichte nämlich,

- ob und wann vor der Kündigung eine unternehmerische Entscheidung tatsächlich getroffen wurde und,

- ob die behauptete unternehmerische Entscheidung bei ihrer Umsetzung tatsächlich dazu führt, dass der Beschäftigungsbedarf für den gekündigten Arbeitnehmer entfällt.

Hierzu muss der Arbeitgeber dem Arbeitsgericht die dafür erforderlichen Daten nachvollziehbar offenlegen. Weder der allgemeine Beschluss, Personalkosten zu senken, noch ein Verweis auf rückläufige Finanzkennzahlen (diese besagen nämlich noch nichts über die
Veränderung der zu erledigenden Arbeitsmenge) reichen dafür aus. Gleiches gilt für den Verweis auf den Abbau einer Hierarchieebene oder den Hinweis auf eine künftige Entwicklung, ohne dass die betrieblichen Umstände konkrete und greifbare Formen angenommen hätten.

\section{Anhörung des Betriebsrats erforderlich}

Häufig wird zudem übersehen, dass in Kliniken vor jeder Kündigung auch der Betriebsrat gemäß $§ 102$ Abs. 1 Satz 1 Betriebsverfassungsgesetz anzuhören ist. Nach der ständigen Rechtsprechung des Bundesarbeitsgerichts ist eine Kündigung nämlich nicht nur dann unwirksam, wenn der Arbeitgeber gekündigt hat, ohne den Betriebsrat überhaupt zu beteiligen. Auch wenn der Arbeitgeber den Betriebsrat zwar über die Kündigung unterreichtet, die Sachlage aber nicht vollständig dargelegt hat, ist die Kündigung unwirksam.

Um den Betriebsrat ordnungsgemäß zu informieren, muss der Arbeitgeber diesem die aus seiner Sicht subjektiv tragenden Kündigungsgründe mitteilen. Auch diesbezüglich obliegt dem Arbeitgeber die Beweislast dafür, dass er den Betriebsrat ordnungsgemäß und umfassend aufgeklärt hat.

\section{Fristen bei der Klage beachten}

Eine entsprechende Kündigungsschutzklage muss spätestens 3 Wochen nach Zugang der schriftlichen Kündigung erhoben werden, da die Kündigung andernfalls als wirksam gilt ( $\S \S 4$ und 7 Kündigungsschutzgesetz). Dabei gilt im Rahmen einer Kündigungsschutzklage der Grundsatz, dass jede Partei ihre Kosten selbst trägt.

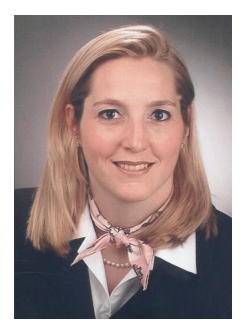

Korrespondenz Dr. iur. Isabel Häser Rechtsanwältin Ehlers, Ehlers \& Partner Widenmayerstraße 29 80538 München i.haeser@eep-law.de 